\title{
Properties of multilayer composite thin films based on morphotropic phase boundary $\mathrm{Pb}\left(\mathrm{Mg}_{1 / 3} \mathrm{Nb}_{2 / 3}\right) \mathrm{O}_{3}-\mathrm{PbTiO}_{3}$
}

\author{
H. El Hosiny Ali a , R. Jiménez a ${ }^{\text {, J. Ricote }}{ }^{\text {a }}$, J. Pérez de la Cruz ${ }^{\text {b }}$, J.R.A. Fernandes ${ }^{\text {b,c }}$, M.L. Calzada a,* \\ a Instituto de Ciencia de Materiales de Madrid, Consejo Superior de Investigaciones Científicas, ICMM-CSIC, Cantoblanco, 28049 Madrid, Spain \\ b Instituto de Engenharia de Sistemas e Computadores do Porto, INESC TEC - INESC, Campus da FEUP, Rua Dr. Roberto Frias, 378. 4200-465 Porto, Portugal \\ c Universidade de Trás-os-Montes e Alto Douro, 5001-801 Vila Real, Portugal
}

\section{A R T I C L E I N F O}

\section{Article history:}

Received 9 March 2012

Received in revised form 24 July 2012

Accepted 26 July 2012

Available online 2 August 2012

\section{Keywords:}

Thin film

Relaxor-ferroelectric

Ferroelectricity

Piezoelectricity

Pyroelectricity

Multilayers

Perovskite

Chemical solution deposition

\begin{abstract}
A B S T R A C T
It has been reported that ferroelectric and piezoelectric properties of $\mathrm{Pb}\left(\mathrm{Mg}_{1 / 3} \mathrm{Nb}_{2 / 3}\right) \mathrm{O}_{3}-\mathrm{PbTiO}_{3}$ (PMNT) thin films, with compositions close to the morphotropic phase boundary (MPB), show lower values than those reported for bulk ceramics with the same composition, which has been attributed to a reduction of the remnant polarization caused by the small size of the grains in the films. An alternative has been proposed to take full advantage of the excellent piezoelectric properties of polycrystalline PMNT in thin film form: a multilayer configuration that uses ferroelectric layers with large remnant polarization, in this case $\mathrm{PbTiO}_{3}$, to generate an internal electric bias within the PMNT layers and, thus, anchor an induced polarization on them, resulting in a consequent large piezoelectric behavior. The detailed study of the properties of these multilayer composite films reveals the complex correlations that arise in these heterostructures, which are key for the design of optimized piezoelectric films based on MPB PMNT.
\end{abstract}

(C) 2012 Elsevier B.V. All rights reserved.

\section{Introduction}

It is well known that the relaxor-ferroelectric $(1-\mathrm{x}) \mathrm{Pb}\left(\mathrm{Mg}_{1 / 3} \mathrm{Nb}_{2 / 3}\right)$ $\mathrm{O}_{3}-\mathrm{xPbTiO}_{3}$ (PMNT) solid solution shows giant piezoelectric coefficients for compositions in the proximity of the morphotropic phase boundary (MPB) between rhombohedral and tetragonal phases: single crystals of these compositions have $\mathrm{d}_{33}$ piezoelectric coefficients of $\sim 2500 \mathrm{pm} / \mathrm{V}$ [1]. Therefore, they are excellent candidates for their use as piezoelectric transducers. The progressive miniaturization of the devices leads to the preparation of PMNT in thin film form for their integration in microelectronic devices [2-5]. However, in thin film form, it has been observed that the dielectric permittivity values $\left(\varepsilon_{33}\right)$ and the functional properties of the PMNT system are lower than those reported for bulk materials [2]. The reported $d_{33}$ coefficients of PMNT thick films $(1500 \mathrm{~nm})$ with compositions close to the MPB are of $197 \mathrm{pC} / \mathrm{N}$, and decreasing quickly with the reduction of the film thickness down to values of $\mathrm{d}_{33} \sim 42 \mathrm{pC} / \mathrm{N}$ [2] or $\sim 55 \mathrm{pC} / \mathrm{N}$ [3] for films between 200 and $400 \mathrm{~nm}$ thick, which has been attributed to grain size effects [4]. Nevertheless, in the ferroelectric response, the polarization saturation values remain close to those obtained in bulk ceramics [2,5], which implies

\footnotetext{
* Corresponding author. Tel.: + 34 913349062; fax: + 34913720623.

E-mail address: lcalzada@icmm.csic.es (M.L. Calzada).
}

that the problem relies on the decrease of the remnant polarization $\left(P_{r}\right)$ values in the thin films.

Based on the principles traditionally used in bulk composites [6], the packing of layers of different ferroelectric compositions in a multilayer composite (MLC) configuration has proved to improve the dielectric and ferroelectric properties, when compared to those corresponding to single phase films $[7,8]$. Several multilayered ferroelectric films and superlattices have been designed searching stress or strain effects, not only due to epitaxial relations [9], but also in polycrystalline heterostructures [10]. Or a simply combination of the properties of the constituent phases is sought, which results in dielectric and ferroelectric properties different from the individual phases, like for example in the case of $\mathrm{Sr}_{0.8} \mathrm{Bi}_{2.2} \mathrm{Ta}_{2} \mathrm{O}_{9}$ and $\mathrm{SrBi}_{2} \mathrm{Nb}_{2} \mathrm{O}_{9}$ [11] or relaxor phases, $\mathrm{Pb}\left(\mathrm{Zn}_{1 / 3} \mathrm{Nb}_{2 / 3}\right) \mathrm{O}_{3}-\mathrm{BaTiO}_{3}$ and $\mathrm{Pb}\left(\mathrm{Mg}_{1 / 3} \mathrm{Nb}_{2 / 3}\right)$ $\mathrm{O}_{3}[12]$.

Couplings produce product properties that can be very attractive, like for example magnetoelectricity in ferroelectric/magnetostrictive composites. Regarding coupling of the polarization among two phases, it has been reported that a soft $(\mathrm{Pb}, \mathrm{Zr}) \mathrm{TiO}_{3}$ (PZT) with large piezoelectric response is kept in a poled state by a hard PZT, in a 2-2 diphasic composite [13], whose properties as piezoelectric transducer are superior to those of a single-phase ferroelectric. This idea, transferred to piezoelectric films, was used by the authors of this work in a previous paper [14], who have proposed a multilayer composite thin film with 
alternating layers of PMNT (low $\mathrm{P}_{\mathrm{r}}$, but large $\mathrm{P}_{\mathrm{s}}$ ) and $\mathrm{PbTiO}_{3}(\mathrm{PT}$ ) (large $\mathrm{P}_{\mathrm{r}}$, but not large piezoelectric coefficients). The induced polarization on the PMNT layers kept by the neighboring poled PT should result in an improved piezoelectric response of the PMNT based MLC films.

However, our previous work revealed the importance of factors that play an important role in the properties of the MLC films and must be considered in order to optimize their response. The small grain size obtained due to the low thickness of the individual layers deposited was a handicap due to size effects on the ferroelectric behavior of the layers. Besides, in order to establish the coupling among phases as the origin of the improved $\mathrm{P}_{\mathrm{r}}$ values, the existence of a direct correlation between the relative amount of phases and net polarization of the composite film must be discarded. The comparison of MLC films with different amounts of the two phases allows also the discussion on the role of the dielectric permittivity of the phases, as we are studying effects related to the application of an electric field. This work explores these questions on MLC films where the thickness of the layers has been increased in order to avoid deleterious size effects, and different combinations of the layers are used. Not only ferroelectric response of the MLC films is studied, but also other functional properties are analyzed to obtain a complete picture of the behavior of these materials. From this study, the mechanisms leading to the enhancement of the functionality of these MLC films compared with the single phase films are discussed.

\section{Experimental procedure}

$\mathrm{PbTiO}_{3}$ (PT) and $0.65 \mathrm{~Pb}\left(\mathrm{Mg}_{1 / 3} \mathrm{Nb}_{2 / 3}\right) \mathrm{O}_{3}-0.35 \mathrm{PbTiO}_{3}$ (PMNT) precursor sols were prepared, as reported elsewhere [15] and diluted in 1,3-propanediol $\left(\mathrm{OH}-\left(\mathrm{CH}_{2}\right)_{3}-\mathrm{OH}\right)$ in order to reach a 0.3 molar solution. Successive layers of the diluted PT sol were spin-coated onto (111) $\mathrm{Pt} / \mathrm{TiO}_{2} / \mathrm{SiO}_{2} /(100) \mathrm{Si}$ substrates, dried at $350{ }^{\circ} \mathrm{C}$ for $60 \mathrm{~s}$ and crystallized by Rapid Thermal Processing (RTP) at $650{ }^{\circ} \mathrm{C}$ for $6 \mathrm{~s}$, in oxygen and with a heating rate of $200{ }^{\circ} \mathrm{C} \cdot \mathrm{s}^{-1}$. The same deposition conditions and drying and crystallization processes were used for the preparation of PMNT thin films. The multilayer composite thin films (MLC) were fabricated by the deposition, drying and crystallization of seven alternating layers of PT and PMNT, starting and finishing either with a PMNT layer (MLC-PMNT) or with a PT layer (MLC-PT), in order to compare two configurations in which either every PMNT layer is sandwiched between two PT layers or, conversely, every PT layer is placed between two PMNT layers. Therefore, we got two multilayer composite films with different PT volume fractions $\left(\mathrm{V}_{\mathrm{PT}}\right), 0.43$ for MLC-PMNT and 0.57 for MLC-PT.

The crystalline phases developed in the films were monitored by X-ray diffraction (XRD) with Bragg-Brentano geometry. A Siemens D500 powder diffractometer equipped with a $\mathrm{Cu}$ anode was used to perform these measurements. Experimental diffraction patterns were compared with standard X-ray patterns of $\mathrm{PbTiO}_{3}$ (JCPDSICDD 6-452) and $\mathrm{Pb}\left(\mathrm{Mg}_{0.33} \mathrm{Nb}_{0.67}\right) \mathrm{O}_{3}$ (JCPDSICDD 27-1199) perovskite phases. Deconvolution and separation of reflections in the experimental patterns were carried out by using the profile program V1-40 and two pseudo-Voigt functions. Grain size and film thickness were estimated from the Scanning Electron Microscopy images, obtained by a ISIDS-130 field emission gun microscope (FEG-SEM), working at $2 \mathrm{keV}$ and $3 \mathrm{keV}$ for the cross-section and plan-view images, respectively.

In order to carry out conductive, dielectric and ferroelectric measurements, Pt electrodes with different sizes were deposited at room temperature on the films surface by using shadow masks and a BAL-TEC SCD 050 RF sputtering equipment. Capacitors were subjected to a RTP post-annealing at $350{ }^{\circ} \mathrm{C}$ for $600 \mathrm{~s}$ in an oxygen atmosphere. The dielectric measurements were carried out at room temperature by a HP 4284A precision LCR meter using a $0.01 \mathrm{~V}$ at $1 \mathrm{kHz}$ driving voltage. Ferroelectric hysteresis loops were measured with a home built system, consisting in a virtual ground circuit. In this system, a HP $8116 \mathrm{~A}$ pulse generator was used to apply voltage sinusoidal waves at a frequency of $1 \mathrm{kHz}$ and the loops were traced by a Tektronix TDS520 oscilloscope. All non-switching contributions were eliminated by a fitting procedure based on a model that simulates the linear part of these contributions by introducing the capacitance and resistance of the films [16]. Non-linear contributions, leakage currents, are fitted by a model that considers the electrical current proportional to $\mathrm{V}^{2}$ [17]. Alternatively, a commercial RT66A standardized ferroelectric test system at a frequency of $1 \mathrm{kHz}$ was also used for measuring hysteresis loops, where a delay time of $1 \mathrm{~s}$ between the voltage conditioning and the measuring triangular waves was applied. This allows us to observe the time relaxation of the polarization, by the comparison of the initial and final polarization values of the loops.

The macroscopic piezoelectric hysteresis loops were carried out by using a fiber optic, double-beam incidence Mach-Zehnder interferometer, as reported elsewhere [18]. The measurements were performed using an AC driving voltage of $5 \mathrm{~V}$ at $7.5 \mathrm{kHz}$, while the DC electric field was changed from 100 to $200 \mathrm{kV} \cdot \mathrm{cm}^{-1}$. The local piezoelectric characterization was carried out with a commercial scanning force microscope (Nanotec ${ }^{\circledR}$ Electrónica, controlled by WSxM ${ }^{\circledR}$ software [19]), which piezoresponse force microscopy (PFM) has been implemented in. Conductive Pt/Ir coated tips (Nanosensors) on cantilevers with a force constant of $42 \mathrm{~N} \cdot \mathrm{m}^{-1}$ were used, with an applied AC voltage of $1 \mathrm{~V}$ at $50 \mathrm{kHz}$. In-field local piezoelectric hysteresis loops have been measured at different locations of the studied films.

The evolution of the pyroelectric coefficient $(\gamma)$ with time, before and after poling, allowed us to evaluate the retention of the polarization of the PMNT and MLC films. The pyroelectric measurements were performed using a dynamic method [20,21]. Triangular thermal waves with $2 \mathrm{~K}$ of amplitude and frequency of $2 \cdot 10^{-3} \mathrm{~Hz}$ were used, around a temperature of $308 \mathrm{~K}$. The thermo-stimulated current obtained was described by the equation:

$I_{p y r}=\mathrm{A} \cdot \gamma \cdot \mathrm{dT} / \mathrm{dt}$

where $A$ is the area of the capacitor and $\gamma$ is the pyroelectric coefficient.

\section{Experimental results}

Fig. 1 shows the XRD patterns of the PT, PMNT and MLC thin films, where no secondary crystalline phases are detected in any of them. The PT and PMNT phases can be identified for the two MLC films, indicating that no significant interdiffusion between layers is produced. As the diffraction peaks of these two phases are usually too close, details of the patterns are shown in Fig. 1(a) to (d), where the solid lines correspond to the deconvolution of the experimental data into the 100 and 200 reflections of the PT and PMNT perovskite phases, providing additional evidence to the absence of other crystalline phases than those of the composite constituent layers.

The single phase PMNT film presents larger lateral grain size than the MLC films: $270 \mathrm{~nm}$ (Fig. 2(a)). By contrast, similar lateral grain size of the superficial PMNT and PT layers is observed in the FEG-SEM images of the MLC film surfaces, 130 and $100 \mathrm{~nm}$, respectively (Fig. 2(b) and (c)). This can be attributed to the grain growth limitation imposed by the thickness of the alternating layers of both phases (around 35-40 nm) in the MLC films. Cross-section SEM images reveal that after seven deposited layers the total thickness of the MLC-PMNT and MLC-PT are $255 \mathrm{~nm}$ and $294 \mathrm{~nm}$, respectively (see Fig. 2(b) and (c)). In single phase films, the grains can grow through the whole thickness of the film, leading to the columnar grain growth of the PMNT film, with thickness of $274 \mathrm{~nm}$ (see Fig. 2(a)). As a result, grains forming the MLC films show equivalent sizes in thickness and laterally. This is a condition that must be considered when the electrical behavior of the MLC films is compared to that observed in the PMNT single phase film.

The piezoresponse force microscopy images (Fig. 3) show that the films have strong self-polarization. The $\mathrm{d}_{33}^{\text {eff }}$ images do not show any trace of ferroelectric domains inside the grains of any of the studied 

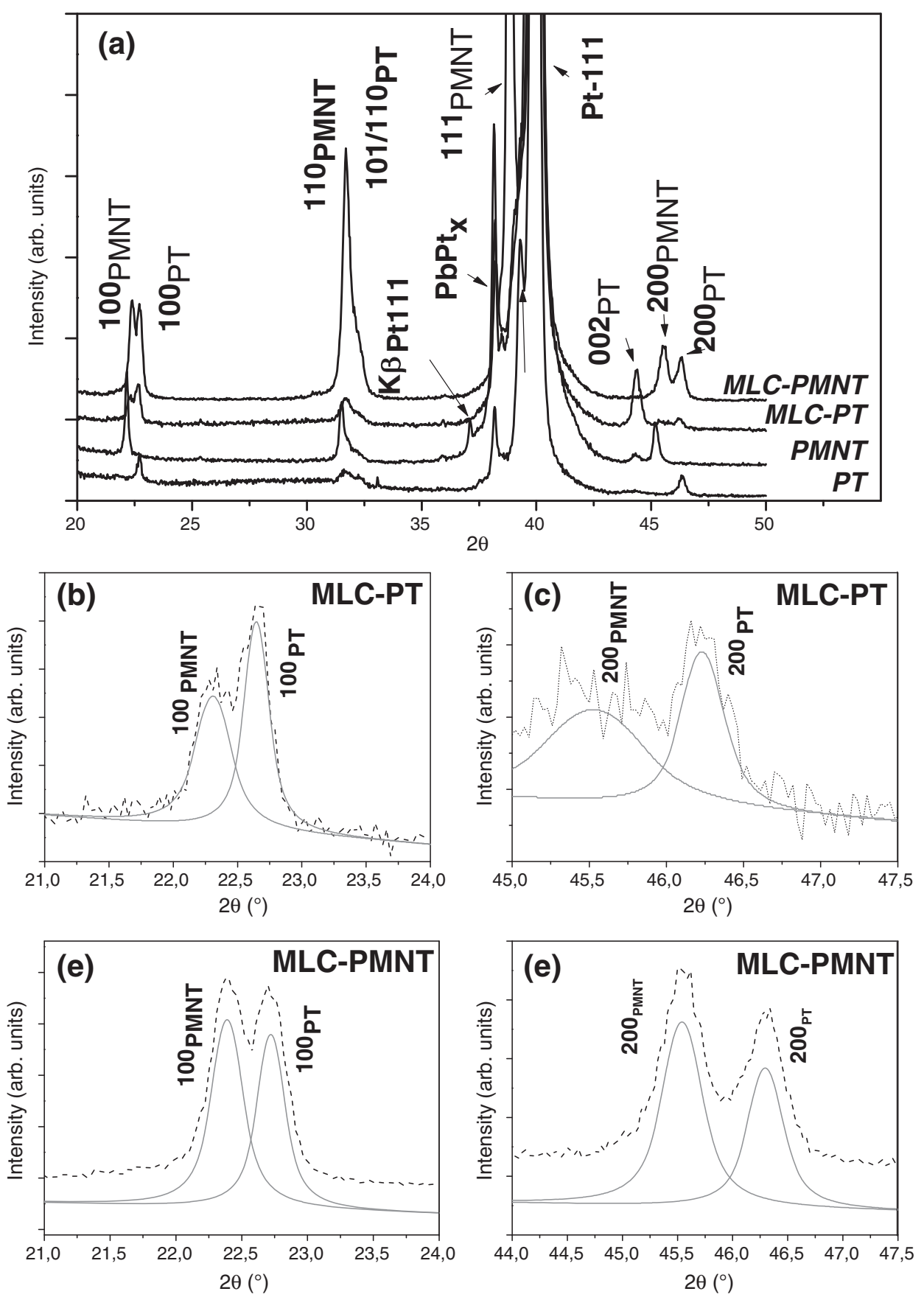

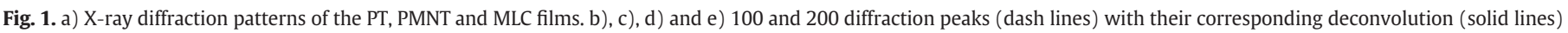
calculated for the PT and PMNT layers.

films. Thus, in this case the size variations seem not to be large enough to produce significant changes in the ferroelectric domain configurations.

The corrected ferroelectric hysteresis loops of the PMNT and MLC films are shown in Fig. 4(a). As expected, the PMNT film have a slanted loop, with saturation polarization $2 \mathrm{P}_{\mathrm{s}}$ of $26 \mu \mathrm{C} \cdot \mathrm{cm}^{-2}$ and remnant values $2 \mathrm{P}_{\mathrm{r}}$ of $9 \mu \mathrm{C} \cdot \mathrm{cm}^{-2}$. These values are similar to those of the MLC-PMNT film, with $2 \mathrm{P}_{\mathrm{s}}$ of $26 \mu \mathrm{C} \cdot \mathrm{cm}^{-2}$ and $2 \mathrm{P}_{\mathrm{r}}$ of $12 \mu \mathrm{C} \cdot \mathrm{cm}^{-2}$; however, a significant change is observed in the coercive field, $2 \mathrm{E}_{\mathrm{C}}$, of this sample, which increases to $240 \mathrm{kV} \cdot \mathrm{cm}^{-1}$. In the MLC-PT sample, where all the PMNT layers are sandwiched between two poled PT layers, both, saturation and remnant polarizations, are twice higher than those of the PMNT film, with $2 \mathrm{P}_{\mathrm{s}}$ of $44 \mu \mathrm{C} \cdot \mathrm{cm}^{-2}$ and $2 \mathrm{P}_{\mathrm{r}}$ of $22 \mu \mathrm{C} \cdot \mathrm{cm}^{-2}$, while $2 \mathrm{E}_{\mathrm{C}}$ is $240 \mathrm{kV} \cdot \mathrm{cm}^{-1}$. A negative bias field is observed for the three samples.
The time relaxation of the polarization is observed in the ferroelectric hysteresis loops obtained with a commercial RT66A Radiant equipment (Fig. 4(b)), as explained before. This reaches a value of more than $80 \%$ loss of remnant polarization ( $1 \mathrm{~s}$ of relaxation or delay time) for the PMNT film (from $6 \mu \mathrm{C} \cdot \mathrm{cm}^{-2}$ to $1 \mu \mathrm{C} \cdot \mathrm{cm}^{-2}$ ), which is still high for the MLC-PMNT film: $50 \%$ loss (from $10 \mu \mathrm{C} \cdot \mathrm{cm}^{-2}$ to $6 \mu \mathrm{C} \cdot \mathrm{cm}^{-2}$ ). This fast relaxation almost disappears for the MLC-PT film, showing the effectiveness of this configuration to retain the polarization.

The retention of the remnant polarization for a long time is one of the most important factors to demonstrate the functional reliability of the MLC films. There are many ways to perform this characterization; however, one of the less perturbing is the measurement of the evolution of the pyroelectric coefficient $(\gamma)$ with time, before and after poling $[20,21]$. The evolution with time of the pyroelectric coefficient for 

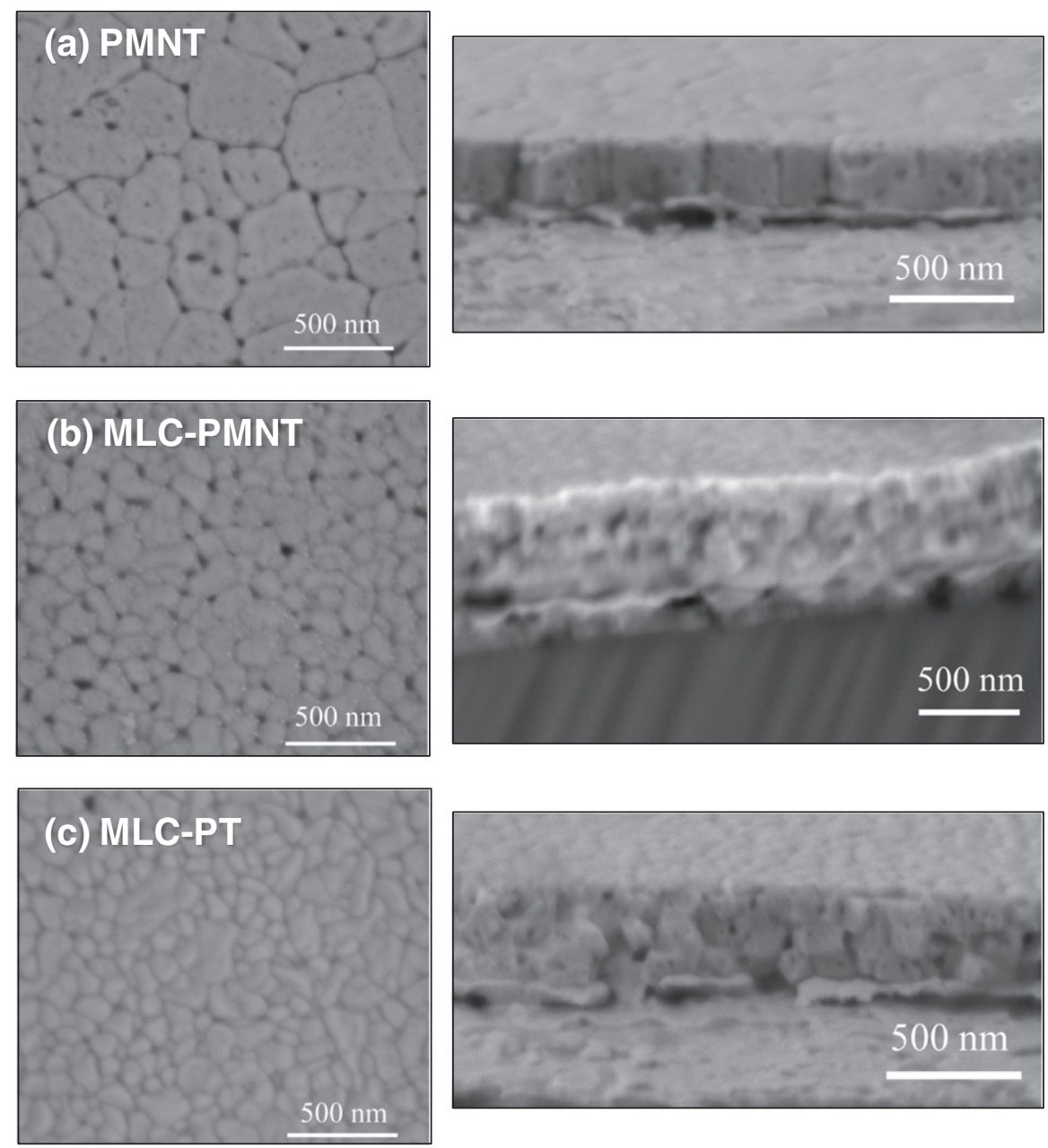

Fig. 2. Plan view and cross-section FEG-SEM images of: a) PMNT, b) MLC-PMNT and c) MLC-PT films.

unpoled and poled films with $300 \mathrm{kV} \cdot \mathrm{cm}^{-1}$ is shown in Fig. 5. Poling is carried out at room temperature with a train of square pulses of $10 \mu \mathrm{s}$ of duration and $300 \mathrm{kV} \cdot \mathrm{cm}^{-1}$ of amplitude, both upwards (i.e. towards the film surface, positive sign) and downwards (i.e. towards the film surface, negative sign). This procedure assures at least $90 \%$ of polarization. The non-zero values of $\gamma$ obtained for all the films before the poling process indicate that all of them show self-polarization. It is observed that the PMNT film suffers an $\sim 88 \%$ loss of the initial $\gamma$ value after $12 \mathrm{~h}$, when it is poled against its self-polarization (Fig. 5(a)). For MLC-PMNT films, the poling process produces rather stable values of the pyroelectric coefficient with time (Fig. 5(b)), besides the significant increase of the overall values. The pyroelectric coefficient of the MLC-PT film only decreases an $\sim 27 \%$ for a long time (Fig. 5(c)). These results show an enhancement of the stability of the polarization in the MLC films, in addition to a significant increase of the coefficient values.

Fig. 6 shows the macroscopic piezoelectric hysteresis loops measured by optical interferometry. Similar to the ferroelectric hysteresis loops already discussed, the PMNT film shows a slim piezoelectric loop, with a value of the piezoelectric coefficient $2\left(\mathrm{~d}_{33}^{\text {eff }}\right)_{\mathrm{r}}$ of $115 \mathrm{pm} \cdot \mathrm{V}^{-1}$. However, the comparison of this value with those obtained for the multilayer films is not possible, because their piezoelectric loops are not saturated. They are most probably sub-coercive loops, needing the application of higher electric fields. It has been shown that coercive fields for the MLC films are larger than for PMNT, being their electrical stability under a static field lower. This does not allow the application of large electric fields for a long time, without the appearance of leakage currents. The stepwise application of the
DC field for these piezoelectric measurements restricts the use of large electric fields for the multilayer films. As a consequence, the piezoelectric behavior of the MLC films cannot be properly compared with that of the PMNT film, although this technique provides quantitative values of the piezoelectric coefficients.

The quantification of the piezoelectric coefficients is difficult by PFM, however, it is possible the local application of large electric fields. Fig. 7 shows local in-field piezoresponse loops that have reached saturation for the three films studied in this work. An enhancement of the remnant piezoelectric coefficients of the MLC films is observed, mainly for the MLC-PT film. The MLC films show a fairly constant value when saturation is reached. On the contrary, the piezoelectric coefficient of the PMNT film always increases with the electric field. Note that the initial and final values of the loops are different for the PMNT and MLC-PMNT films, similar to those of the RT66A hysteresis loops. This is because both measurements are carried out with a lapse of time under a short circuit condition among the successive measurements.

\section{Discussion}

The results of the previous section show an increase of the polarization values in the multilayer composite films with respect to the single phase PMNT film. However, this cannot be only explained by the linear additive effect obtained from the equation:

$$
\mathrm{P}_{\mathrm{r}}^{\mathrm{MLC}}=\left(\mathrm{V}_{\mathrm{PT}}\right)^{\mathrm{MLC}} \cdot \mathrm{P}_{\mathrm{r}}^{\mathrm{PT}}+\left(\mathrm{V}_{\mathrm{PMNT}}\right)^{\mathrm{MLC}} \cdot \mathrm{P}_{\mathrm{r}}^{\mathrm{PMNT}}
$$



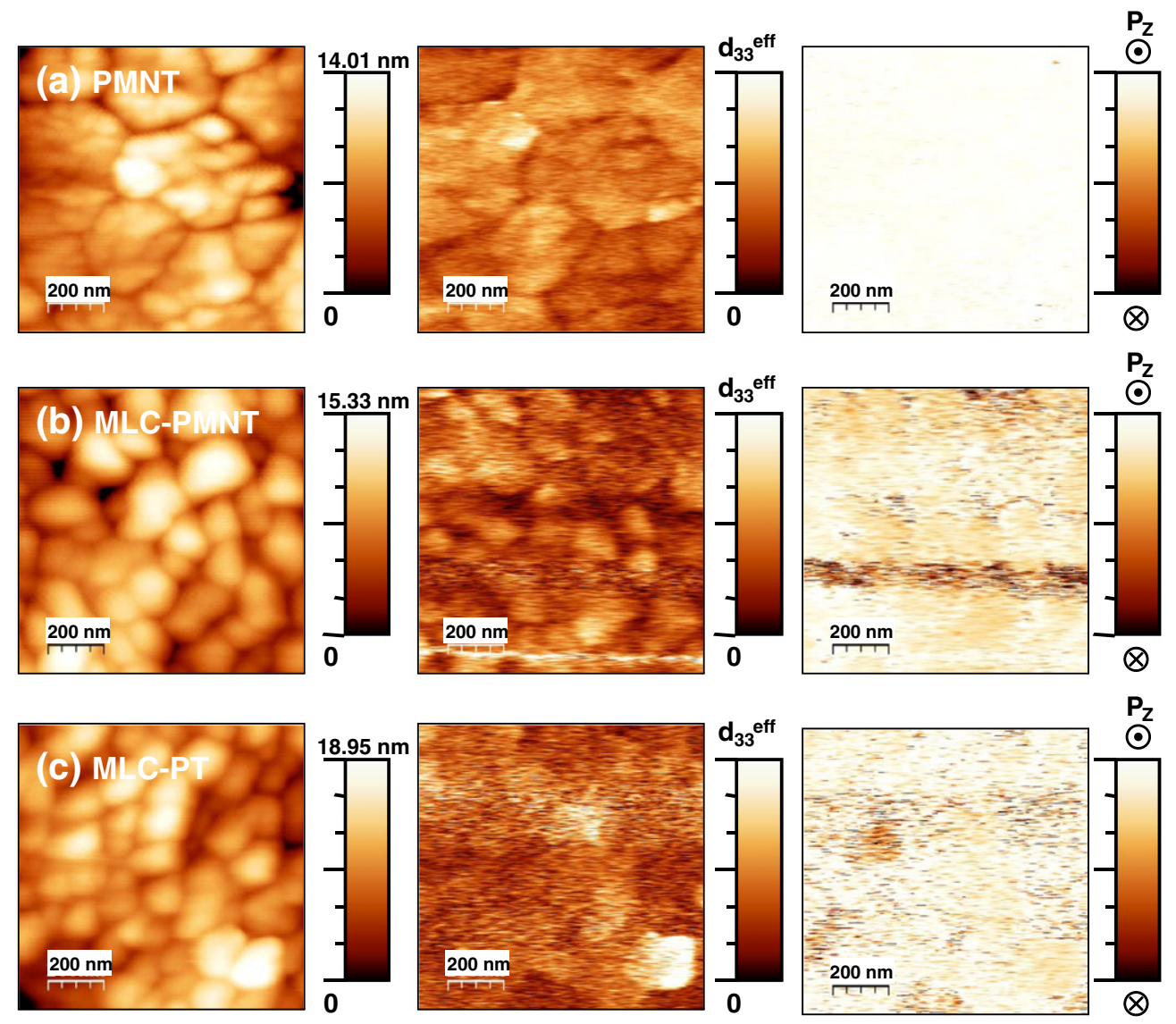

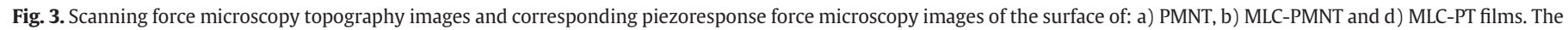
scales for $\mathrm{d}_{33}^{\text {eff }}$ are different for the three films and are adjusted for a better visualization of the results.

where $\mathrm{V}_{\mathrm{PT}}$ and $\mathrm{V}_{\mathrm{PMNT}}$ are the volume fractions of PT and PMNT in the MLC films, and the experimental $\mathrm{P}_{\mathrm{r}}$ values of the PT and PMNT single phase films are used; $2 \mathrm{P}_{\mathrm{r}}^{\mathrm{PT}} \sim 60 \mu \mathrm{C} \cdot \mathrm{cm}^{2}$ and $2 \mathrm{P}_{\mathrm{r}}^{\mathrm{PMNT}} \sim 9 \mu \mathrm{C} \cdot \mathrm{cm}^{-2}$.

Taking into account the much higher values of $\mathrm{P}_{\mathrm{r}}^{\mathrm{PT}}$ than $\mathrm{P}_{\mathrm{r}}^{\mathrm{PMNT}}$, the PT phase should dominate the $\mathrm{P}_{\mathrm{r}}^{\mathrm{MLC}}$ values in the multilayer composite films, being the $P_{r}^{M L C-P T} / P_{r}^{M L C-P M N T}$ ratio close to the volume fraction of PT, for each of the composite films here designed, $\left(\mathrm{V}_{\mathrm{PT}}\right)^{\mathrm{MLC}-\mathrm{PT}} /\left(\mathrm{V}_{\mathrm{PT}}\right)^{\mathrm{MLC}-\mathrm{PMNT}}=$ 1.33.

$\mathrm{P}_{\mathrm{r}}^{\mathrm{MLC}-\mathrm{PT}},\left(\mathrm{V}_{\mathrm{PT}}\right)^{\mathrm{MLC}-\mathrm{PT}}$, and $\mathrm{P}_{\mathrm{r}}^{\mathrm{MLC}-\mathrm{PMNT}},\left(\mathrm{V}_{\mathrm{PT}}\right)^{\mathrm{MLC}-\mathrm{PMNT}}$ are the remnant polarization and volume fraction of PT in the MLC-PT and MLC-PMNT films, respectively. Indeed, the $2 \mathrm{P}_{\mathrm{r}}$ values for the MLC-PMNT and MLC-PT films calculated from Eq. (2) are $31 \mu \mathrm{C} \cdot \mathrm{cm}^{-2}$ and $38 \mu \mathrm{C} \cdot \mathrm{cm}^{-2}$, respectively, obtaining a calculated $\left(\mathrm{P}_{\mathrm{r}}^{\mathrm{MLC}-\mathrm{PT}} / \mathrm{P}_{\mathrm{r}}^{\mathrm{MLC}-\mathrm{PMNT}}\right)_{\text {calc }}$ ratio of 1.23 , close to the PT volume ratio of 1.33 . This is an approximation, due to the very different characteristics of the single phase PMNT and MLC films. The PMNT film has columnar grains with a considerable crystal texture, whereas the MLC films show a polcrystaline grain growth (see Figs. 1 and 2). In spite of this, a large difference is observed between the $\left(\mathrm{P}_{\mathrm{r}}^{\mathrm{MLC}-\mathrm{PT}} / \mathrm{P}_{\mathrm{r}}^{\mathrm{MLC}-\mathrm{PMNT}}\right)_{\text {calc }}$ ratio of 1.23 and that obtained from the experimental values of $P_{r}$, $\left(\mathrm{P}_{\mathrm{r}}^{\mathrm{MLC}-\mathrm{PT}} / \mathrm{P}_{\mathrm{r}}^{\mathrm{MLC}-\mathrm{PMNT}}\right)_{\exp } \sim 1.83$. This indicates that other mechanisms, different from the linear addition of the properties of the constituent phases, have to be considered in these MLC films.

Note that Eq. (2) is valid for static conditions (a time large enough for reaching the saturation of the polarization in each of the layers: full poling switching). This is not the case of the ferroelectric hysteresis loops of Fig. 4(a), carried out at $1 \mathrm{kHz}$ for $1 \mathrm{~ms}$. Under these conditions, the switching kinetics seem to be different for the MLC-PT and MLC-PMNT films, as inferred from the more or less slanted shape of the loops (see Fig. 4(a)). The switching behavior of the PT layers does not make possible to retain a high value of the polarization at zero field, thus yielding to a poor remnant polarization in the MLC-PMNT film. The most favorable switching kinetics of the MLC-PT film lead to an improved polarization of the PT layer, resulting in much higher values of the remnant polarization.

Note the results obtained at zero field after a relaxation time of $1 \mathrm{~s}$ in the ferroelectric hysteresis loops of Fig. 4(b). A relatively large and fast relaxation of the polarization is observed in the MLC-PMNT film, while this is negligible for the MLC-PT film. This supports the good retention of the polarization of the MLC-PT film, indicating that the polarization in the PMNT layers induced by the poled PT ones is clearly much more effective when each of the former is sandwiched between two of the latter.

The retention of the polarization has been studied by means of the evolution of the pyroelectric coefficient for a long time (Fig. 5). The PMNT films have a strong relaxation of the polarization for a very short time, when poling is carried out against its self-polarization; only $25 \%$ of the initial value is retained after the withdrawal of the poling field. Polarization is more stable when the electric field is applied in the same sense as its self-polarization vector. This is the regular behavior of polycrystalline PMNT films, as previously reported [4]. The MLC films have higher values of the pyroelectric coefficient than the PMNT film, which is associated with the enhancement of the polarization in the composite multilayer configuration discussed above. In addition, the asymmetry in the polarization disappears. The MLC-PMNT film shows stable values of the pyroelectric coefficient with time, after the initial decrease for a short time, observed in the P-E loops of Fig. 4(b). The MLC-PT film has a slow relaxation of the coefficient values with time (Fig. 5(c)), with a typical behavior of a ferroelectric film [20]. Therefore, it can be concluded that the combination of PMNT and PT layers in a 2-2 composite thin film, not only results in an increase of the remnant values of the functional properties of the resulting PMNT-based films, but also in a long 

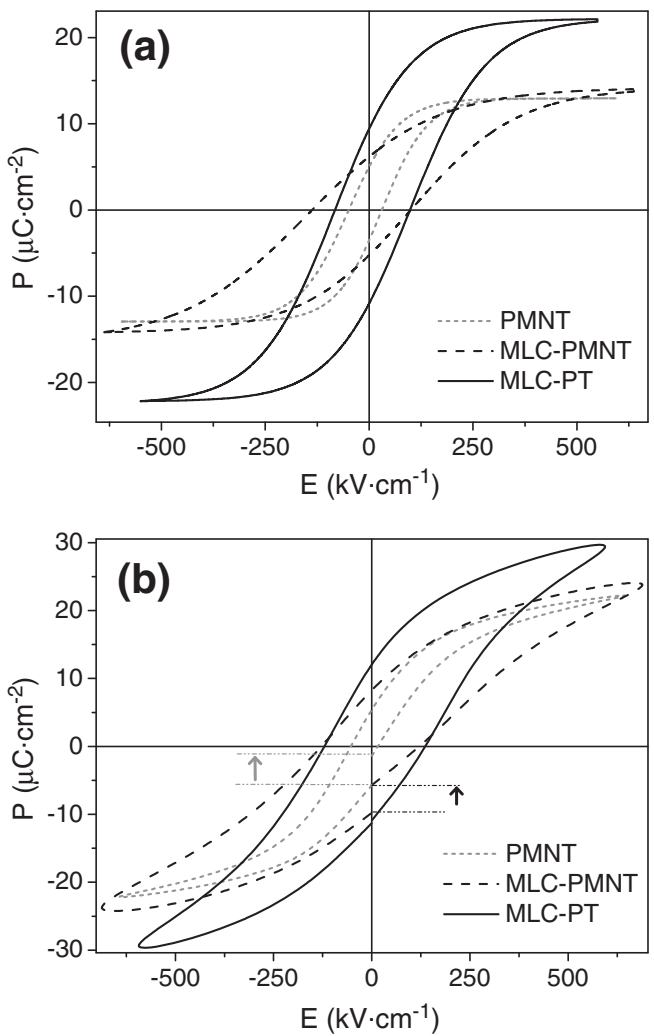

Fig. 4. Ferroelectric hysteresis loops of the PMNT and MLC films measured at $1 \mathrm{kHz}$ using (a) a home built system (non-switching and leakage current contributions removed) and (b) commercial RT66A Radiant equipment.

stability of the properties, surpassing the reliability problems associated to relaxor-ferroelectric thin films. This cannot be explained by a simple additive effect of the properties of the phases forming the MLC films, but by the polarization induced and stabilized in the PMNT layers of the composite films by the poled PT layers.

Regarding the piezoelectric response of the MLC films, the effective remnant longitudinal piezoelectric coefficient of a biphasic multilayer composite, $\mathrm{d}^{\mathrm{MLC}}{ }_{33}$, can be obtained from the following equation [13]:

$\mathrm{d}_{33}^{\mathrm{MLC}}=\frac{\left[\mathrm{d}_{33}^{\mathrm{PT}} \cdot \varepsilon_{33}^{\mathrm{PMNT}} \cdot\left(\mathrm{V}_{\mathrm{PT}}\right)^{\mathrm{MLC}}+\mathrm{d}_{33}^{\mathrm{PMNT}} \cdot \varepsilon_{33}^{\mathrm{PT}} \cdot\left(\mathrm{V}_{\mathrm{PMNT}}\right)^{\mathrm{MLC}}\right]}{\varepsilon_{33}^{\mathrm{PMNT}} \cdot\left(\mathrm{V}_{\mathrm{PT}}\right)^{\mathrm{MLC}}+\varepsilon_{33}^{\mathrm{PT}} \cdot\left(\mathrm{V}_{\mathrm{PMNT}}\right)^{\mathrm{MLC}}}$

where $V_{\text {PT }}$ and $V_{\text {PMNT }}$ are the volume fractions and $\varepsilon_{33}^{\mathrm{PMNT}}$ and $\varepsilon_{33}^{\mathrm{PT}}$ are the dielectric permittivity of the PT and PMNT phases.

Taking into account the remnant polarization after $1 \mathrm{~s}$ of relaxation, obtained from Fig. 4(b), and the dielectric constants measured at room temperature in the single phase films, it can be considered:

$\mathrm{d}_{33}^{\mathrm{PT}} \cdot \delta_{33}^{\mathrm{PMNT}} \cdot\left(\mathrm{V}_{\mathrm{PT}}\right)^{\mathrm{MLC}} \gg \mathrm{d}_{33}^{\mathrm{PMNT}} \cdot \delta_{33}^{\mathrm{PT}} \cdot\left(\mathrm{V}_{\mathrm{PMNT}}\right)^{\mathrm{MLC}}$.

Thus, the effective longitudinal piezoelectric coefficient of the MLC films has to be always higher than the value obtained in the equivalent single phase PT film (considering the PT volume fraction); $\mathrm{d}_{33}^{\mathrm{MLC}} \geq \mathrm{d}_{33}^{\mathrm{PT}}$. $\mathrm{V}_{\mathrm{PT}}$, and depending on the ratio of the dielectric constants of the two phases of the MLC film; $\varepsilon_{33}^{\mathrm{PMNT}} / \varepsilon_{33}^{\mathrm{PT}}$. But, if a large polarization is induced in the PMNT layers of the MLC films by the PT ones, then the $\mathrm{d}_{33}^{\mathrm{MLC}}$ value measured would be considerably much higher than the $\mathrm{d}_{33}^{\mathrm{PT}}$. The macroscopic piezoelectric data of Fig. 6 do not show this effect. Here, the high coercive fields of the MLC films make imperative the application of a large electric field, compared with the PMNT single phase film, which leads to leakage currents in the films and make impracticable the stepwise application of high electric fields. This is surpassed with the
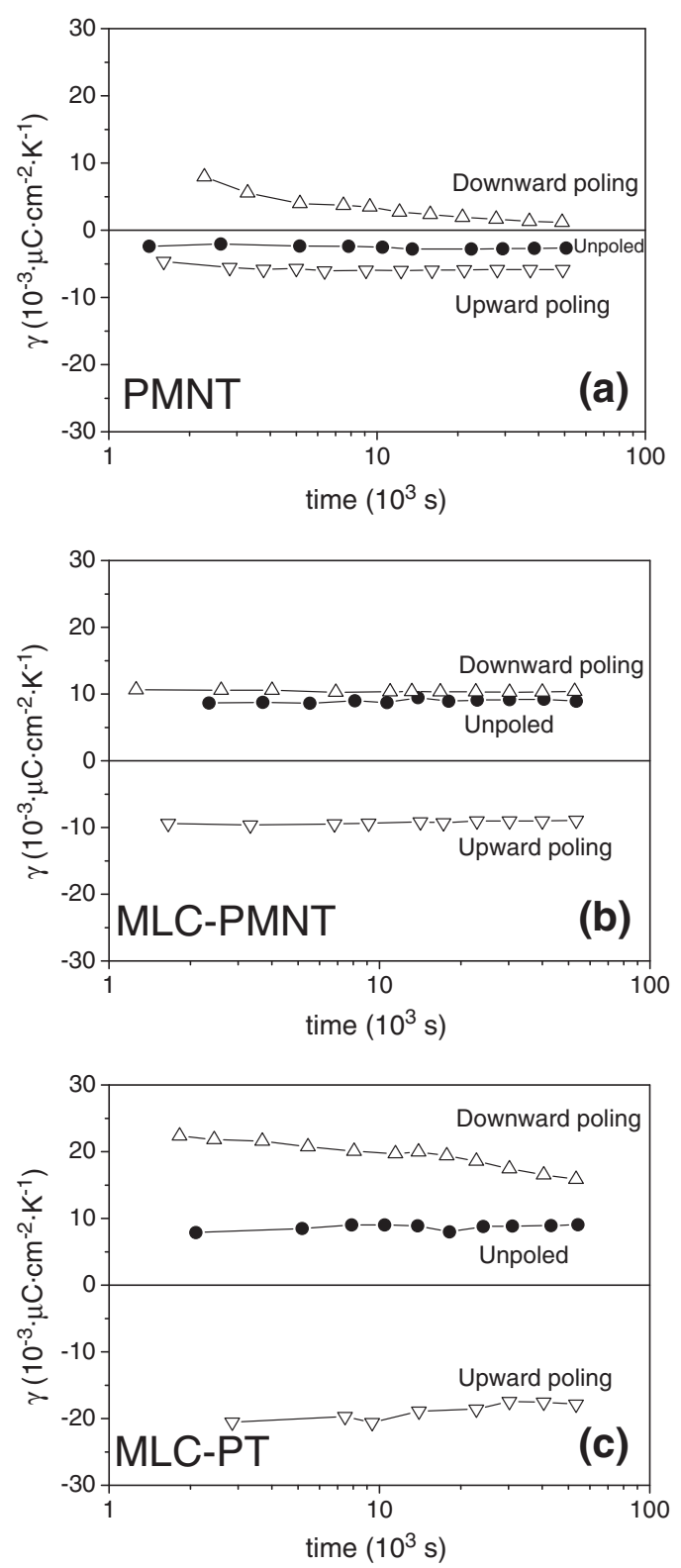

Fig. 5. Evolution with time of the pyroelectric coefficient $(\gamma)$ before and after poling at room temperature with a train of square pulses of $10 \mu \mathrm{s}$ of duration and $300 \mathrm{kV} \cdot \mathrm{cm}^{-1}$ of amplitude, both upwards (i.e. towards the film surface, positive sign) and downwards (i.e. towards the film surface, negative sign) for: a) PMNT, b) MLC-PMNT and c) MLC-PT films.

piezoresponse force microscopy experiments. Although quantitative values of the local piezoelectric coefficients cannot be obtained from these experiments, the piezoresponse results evidence a higher piezoelectric response for the MLC-PT film compare with the single phase PMNT and the MLC-PMNT films (Fig. 7), not observing the characteristic fast relaxation of the relaxor-ferroelectric materials.

Summarizing, the study of the properties of 2-2 biphasic multilayer composite thin films based on the relaxor-ferroelectric $\mathrm{Pb}\left(\mathrm{Mg}_{1 / 3} \mathrm{Nb}_{2 / 3}\right)$ $\mathrm{O}_{3}-\mathrm{PbTiO}_{3}$ (PMNT), with compositions close to the morphotropic phase boundary (MPB), shows that stable polarization can be induced in PMNT layers by neighboring poled ferroelectric layers, like $\mathrm{PbTiO}_{3}$ (PT). This multilayer film configuration is very effective when every PMNT layer is sandwiched between two PT layers. This produces a considerable enhancement of the remnant polarization with respect to the single phase PMNT films, and an improvement of the piezoelectric response of the polycrystalline composite PMNT-based thin films compare with the single phase relaxor-ferroelectric PMNT films. 


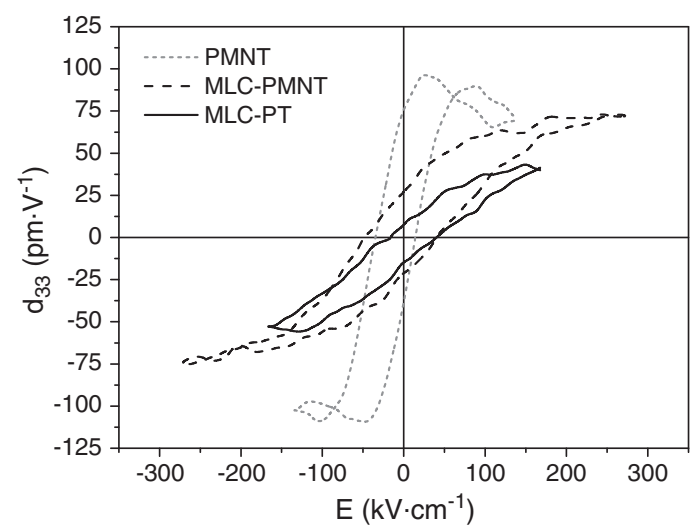

Fig. 6. Macroscopic piezoelectric responses of PMNT and MLC thin films. Measurements were carried out by a fiber optic, double-beam incidence Mach-Zehnder interferometer.

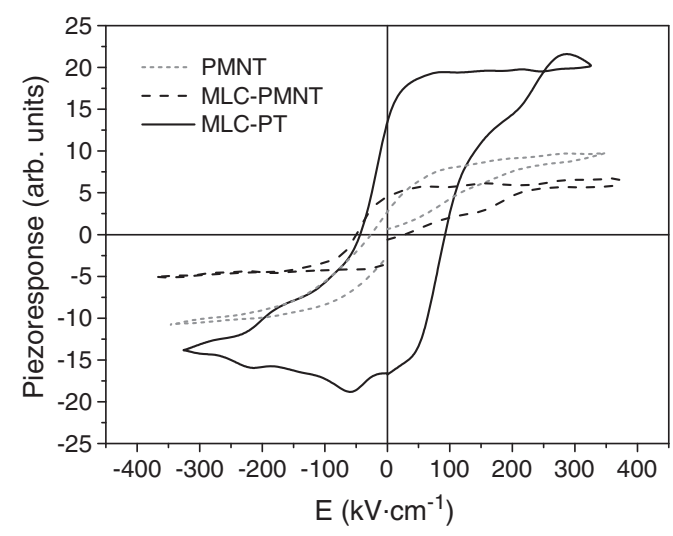

Fig. 7. Local piezoresponse loops of PMNT and MLC thin films.

\section{Acknowledgments}

This work has been funded by the Spanish Project MAT2010-15365. H. El Hosiny thanks the Spanish Ministry of Science and Innovation for his FPI PhD grant BES-2008-010132.

\section{References}

[1] S.E. Park, T.R. Shrout, J. Appl. Phys. 82 (1997) 1804.

[2] J.H. Park, F. Xu, S. Trolier- Mckinstry, J. Appl. Phys. 89 (2001) 032903.

[3] M.L. Calzada, M. Algueró, A. Santos, M. Stewart, M.G. Cain, L. Pardo, J. Mater. Res. 2 (2009) 24.

[4] M. Algueró, M. Stewart, M.G. Cain, P. Ramos, J. Ricote, M.L. Calzada, J. Phys. D: Appl. Phys. 43 (2010) 205401.

[5] J.H. Park, S. Trolier-Mckinstry, J. Mater. Res. 16 (2001) 268.

[6] R.E. Newnham, Annu. Rev. Mater. Sci. 16 (1986) 47.

[7] K.P. Jayadevan, T.Y. Tseng, J. Mater. Sci. Mater. Electron. 13 (2002) 439.

[8] D. Bao, Curr. Opin. Solid State Mater. Sci. 12 (2008) 55.

[9] H.N. Lee, H.M. Christen, M.F. Chisholm, C.M. Rouleau, D.H. Lowndes, Nature 433 (2005) 395.

[10] R. Poyato, M.L. Calzada, L. Pardo, Appl. Phys. Lett. 86 (2005) 042905.

[11] N. Ortega, P. Bhattacharya, R.S. Katiyar, Mater. Sci. Eng. B 130 (2006) 36.

[12] M.H. Corbett, R.M. Bowman, J.M. Gregg, D.T. Foord, Appl. Phys. Lett. 79 (2001) 815.

[13] R.E. Newnham, D.P. Skinner, L.E. Cross, Mater. Res. Bull. 13 (1978) 525.

[14] H.E.L. Hosiny Ali, R. Jiménez, J. Ricote, M. Algueró, M.L. Calzada, Thin Solid Films 519 (2011) 6467.

[15] M.L. Calzada, M. Algueró, J. Ricote, A. Santos, J. Sol-gel Sci. Technol. 42 (2007) 336.

[16] R. Jiménez, C. Alemany, M.L. Calzada, A. González, J. Ricote, J. Mendiola, Appl. Phys. A 75 (2002) 607.

[17] T.P. Juan, S. Chen, J.Y. Lee, J. Appl. Phys. 95 (2004) 3120

[18] J.R. Fernandes, F.A. de Sá, J.L. Santos, E. Joanni, Rev. Sci. Instrum. 73 (2002) 2073.

[19] I. Horcas, R. Fernández, J.M. Gómez-Rodríguez, J. Colchero, J. Gómez-Herrero, A.M. Baró, Rev. Sci. Instrum. 78 (2007) 0130705.

[20] R. Jiménez, P. Ramos, M.L. Calzada, J. Mendiola, Bol. Soc. Esp. Ceram. Vidrio 37 (1998) 117.

[21] R. Jiménez, B. Jiménez, in: L. Pardo, J. Ricote (Eds.). R. Hull, R.M. Osgood, Jr., J. Parisi, H. Warlimont (Series Eds.), "Pyroelectricity in Polycrystalline Ferroelectrics" in Handbook on Multifunctional Polycrystalline Ferroelectric Materials. Processing and Properties. Springer series in materials science, 140, Springer, 2011, pg. 573. ISBN 978-90-481-2874-7. 